\title{
FEATURES OF DEVELOPMENT OF SUSTAINED FLUXES OF HIGH-ENERGY GAMMA-RAY EMISSION AT DIFFERENT STAGES OF SOLAR FLARES
}

\section{G.S. Minasyants}

Fesenkov Astrophysical Institute,

Almaty, Kazakhstan, gennadii_minasya@mail.ru

T.M. Minasyants

Fesenkov Astrophysical Institute,

Almaty, Kazakhstan, gennadii_minasya@mail.ru

\author{
V.M. Tomozov \\ Institute of Solar-Terrestrial Physics SB RAS, \\ Irkutsk,Russia,tom@iszf.irk.ru
}

\begin{abstract}
We have studied properties of sustained gamma fluxes having quantum energies of $>100 \mathrm{MeV}$ at different stages of flares with 1-min temporal resolution (Fermi/LAT).

The most probable process of emergence of highenergy gamma-quanta during the impulsive phase of flares ( 6 events) has been confirmed. Acceleration of particles, produced by flare energy release (at dissipation of current sheet), occurs when they interact with a shock front of a coronal mass ejection (CME), which develops in the same active region at the same time. Nuclear interactions of accelerated protons $(>500 \mathrm{MeV})$ with plasma ions lead further to the emergence of highenergy gamma-quanta. We have established that the interaction between a flare flux and a high-speed CME during the flare impulsive phase occurs within fairly limited periods - from 2 to $16 \mathrm{~min}$. In the events considered, we have found a direct connection between maximum gamma flux $F_{y>100 \mathrm{Mev}}^{\max }$ and CME velocity.

High maximum values of gamma fluxes are typical of the flare impulsive phase: $3.5 \cdot 10^{-4} \cdot \mathrm{cm}^{-2} \mathrm{~s}^{-1} \leq \leq F_{\gamma>100 \mathrm{MeV}}^{\max }$ $\leq 1.3 \cdot 10^{-2} \cdot \mathrm{cm}^{-2} \mathrm{~s}^{-1}$. At the same time, the value $F_{\gamma>100 \mathrm{Mev}}^{\max }$ $=0.013 \cdot \mathrm{cm}^{-2} \mathrm{~s}^{-1}$ was the highest for the events observed by Fermi/LAT from 2008 to 2017.

During the development of CMEs moving with a
\end{abstract}

supersonic speed, shock waves are formed which are the major power source of accelerated particles during the main phase of gradual flares. In some cases, however, the impact of shock waves on particle acceleration is the greatest in the short impulsive phase.

To reveal parameters most effectively influencing the generation of high-energy gamma-ray emission, we have compared 17 flare events. The most significant parameter proved to be the time interval of joint action of flare process and CME shocks.

We have established that during simultaneous development of flare process and CME attendant on the flare, the most efficient particle acceleration occurs which gives rise to maximum fluxes of high-energy gamma-quanta.

Keywords: flares, coronal mass ejection, particle acceleration, gamma-ray emission.

\section{INTRODUCTION}

Studies of the structure of solar active regions with powerful flares developing in them have identified the most probable sources of particle acceleration such as: electric fields, plasma and MHD turbulence, and shock waves. Each of these sources features duration, efficiency, and typical scale. One of the basic mechanisms of particle acceleration during the flare impulsive phase is thought to be the release of free magnetic energy in the active region due to its dissipation in current sheets caused by magnetic reconnection followed by stochastic acceleration during the development of various plasma instabilities [Altyntsev et al., 1982; Priest, Forbs, 2005; Somov, 2013]. These observations have shown that magnetic reconnection occurs both in the solar corona and in the solar chromosphere [Li et al., 2017]. The second mechanism of particle acceleration in flares (particularly in proton events) is the acceleration at fronts of shock waves generated during propagation of coronal mass ejections (CMEs) from active regions.

According to modern concepts, the occurrence of flares and their associated plasma ejections is closely related to imbalance of magnetic structures of active regions in the course of their evolution. When developing, the magnetic structures of active regions with filaments located on the magnetic field neutral line become more and more complicated. Above the filaments there are closed magnetic field loops, which create a closed magnetic configuration of the entire active region up to coronal heights. Under continuous action of shear movements on footpoints of magnetic loops along the neutral line, a filament (prominence) becomes unstable and starts to rise rapidly, opening the magnetic structure and forming a CME core in the front part. Behind the outgoing CME, a thin luminous "jet" of hot plasma producing a current sheet is formed [Shibata, 1998; Knizhnik et al., 2017]. Let us emphasize that the main contributor to the complication of the magnetic structure are 
considered to be shear movements along the neutral line in the active region [Golovko et al., 1986]. It may be just the breakthrough of apices of coronal magnetic loops by the moving filament that is associated with the flare impulsive phase, because in these very regions energy release and sources of hard X-ray are observed.

It is known that during the flare impulsive phase in the energy release region there are rapidly moving streams of hot plasma and accelerated charged particles, some of which propagate along magnetic flux tubes to underlying layers of the solar atmosphere. When energetic particles interact with denser plasma in footpoints of magnetic loops, X-ray and gamma-ray bremsstrahlung is generated and plasma is heated. The fast heating of plasma in the photosphere and chromosphere causes its evaporation and upwelling with filling of the entire volume of magnetic loops. During this flare phase, the greatest enhancement of soft X-rays occurs. During the flare main phase, the heated plasma in the system of arcs emits in a soft X-ray band for a long time, gradually losing its energy [Livshits, 2008]. At the same time, the flare-induced plasma streams interact with the developing CME. This leads to subsequent acceleration of flare protons at the CME shock front as well as in shock waves driven by CME moving in the upper layers of the corona and in interplanetary space. The occurrence of type II radio bursts in the solar corona and heliosphere is associated with the existence of shock wave fronts in CME. Particles can also be additionally accelerated in the presence of a current sheet formed by magnetic reconnection in the region behind the outgoing CME front [Manchester et al., 2017].

In recent years, much attention has been paid to flares with the sustained gamma-ray emission phase, which are among the most powerful and geoeffective events. They almost always occur with a fast halo CME. Such flares have also been studied before, in particular with the spacecraft (SC) CORONAS-F/SONG [Kurt et al., 2010] and GAMMA/GAMMA1 [Akimov et al., 1991], but little attention has been paid to the role of CME in accelerating protons to high energies. Only individual events in terms of CME have been analyzed in detail [Grechnev et al., 2008].

The flare phase with sustained gamma-ray emission is known to imply a large number of accelerated highenergy particles - protons and electrons interacting with the solar atmosphere. The possibility of nuclear interactions in the solar atmosphere that involves energetic particles in the most powerful flare events accompanied by gamma-ray emission has been raised long ago, particularly for proton events. According to the results obtained by Murphy et al. [1987], protons with energies greater than $300 \mathrm{MeV}$ when interacting with the substance of the solar atmosphere can generate neutral and charged $\pi$-mesons (pions) whose decay produces gamma-ray emission. When flare protons are accelerated to $E_{\mathrm{k}}>500 \mathrm{MeV}$, neutral pions produce high-energy (>100 MeV) gamma-quanta, recorded by SC instruments.

Shock waves during CME are considered to be the main sources of solar protons accelerated to $>500 \mathrm{MeV}$ when they propagate in the solar corona and interplanetary medium [Tylka et al., 2014]. Grechnev et al. [2008] have pointed out that when studying characteristics of sustained events it is very important to assess the relative contribution of particle acceleration in flares and CME shock fronts. This contribution may vary for different events, different stages of their development, and different energy ranges. Gopalswamy et al. [2018, 2019] have found a relationship between characteristics of type II radio bursts and flares with sustained gamma-ray emission, which suggests a significant role of CME shocks in accelerating protons to high energies at large distances in the corona and heliosphere.

An extensive observational material on 30 flares with a long phase of gamma-ray emission obtained with the Large Area Telescope (LAT) of SC Fermi between 2008 and 2017 has been presented and analyzed in [Share et al., $2017 \mathrm{a}, \mathrm{b}]$. In these papers, the period with high-energy (>100 MeV) gamma-quanta was proposed to call the second stable emission stage in the development of powerful flares; the first stage includes the emission during the flare impulsive phase. In such events, the duration of the impulsive phase was less than $20 \mathrm{~min}$, whereas the main phase could last for several hours or more.

By using a new method, Share et al. [2017a, b] from original observational data on gamma fluxes $F_{\gamma>100 \mathrm{MeV}}$ have obtained their values with a higher time resolution (1 min) and with estimated statistical errors.

In this paper, we have analyzed a number of events from the catalog [Share et al., 2017a, b] in order to identify features of the impulsive phase of some flares with sustained gamma-ray emission. We have found 6 events with high-energy (>100 MeV) gamma-quanta during the flare impulsive phase (Section 1). The emphasis is on the comparison of maximum gamma fluxes $F_{\gamma>100 \mathrm{MeV}}^{\max }$ with the parameters that can alter the fluxes in both the impulsive and main phases of the flare and CME (Section 2).

\section{CHARACTERISTICS OF GAMMA- FLUX DEVELOPMENT DURING THE FLARE IMPULSIVE PHASE}

Below are the results of the analysis of features of the evolution of $>100 \mathrm{MeV}$ gamma fluxes during impulsive phases of six flare events.

As already noted, during the flare impulsive phase there are bright areas of downflowing plasma from regions of dissipating current sheets in the corona. SDO photoheliograms in the $\lambda 1700 \AA$ line (images of the upper photosphere and temperature minimum region in the UV spectral range) with high spatial resolution clearly show basic features of this process. Using a specially designed program, we carried out photometric processing of these images and obtained the time profile of the relative intensity $\Delta I$ of the $1700 \AA$ line in photospheric flare plasma (see Figures 1 and 2). The intensity of the pre-flare undisturbed photosphere was taken as 1.00. The temporal resolution for individual measurement series was $\sim 50 \mathrm{~s}$.

The variation of the bright plasma emission intensity in the photosphere in the $1700 \AA$ line is an index that characterizes well the direct effect of energetic particles of flares, which generates hard X-rays and low-energy gamma-quanta [Minasyants et al., 2018]. 


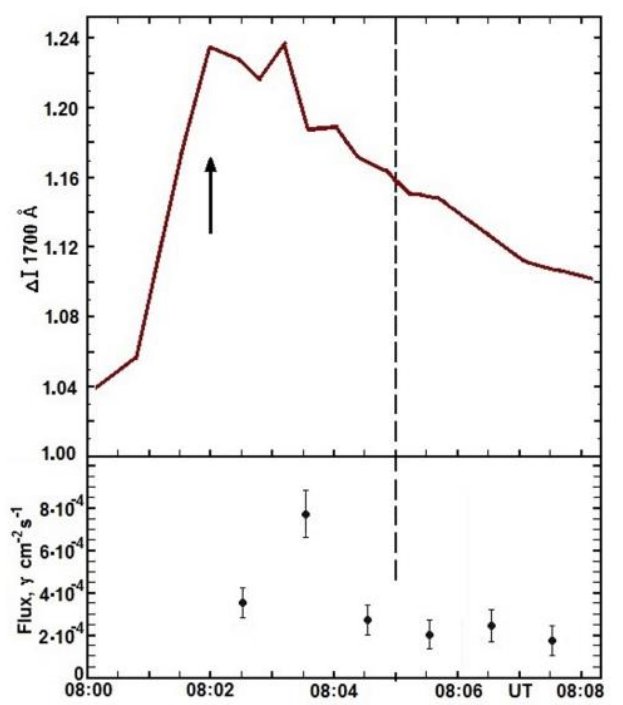

Figure 1. Variation in the relative intensity of photospheric bright emission (top) and $>100 \mathrm{MeV}$ gamma-quanta (bottom) during the August 9, 2011 X6.9 flare (N14, W69). The duration of the flare impulsive phase is 08:00-08:05 UT. The end of the impulsive phase is shown by the vertical dashed line. The vertical arrow marks the onset of CME (08:02 UT)

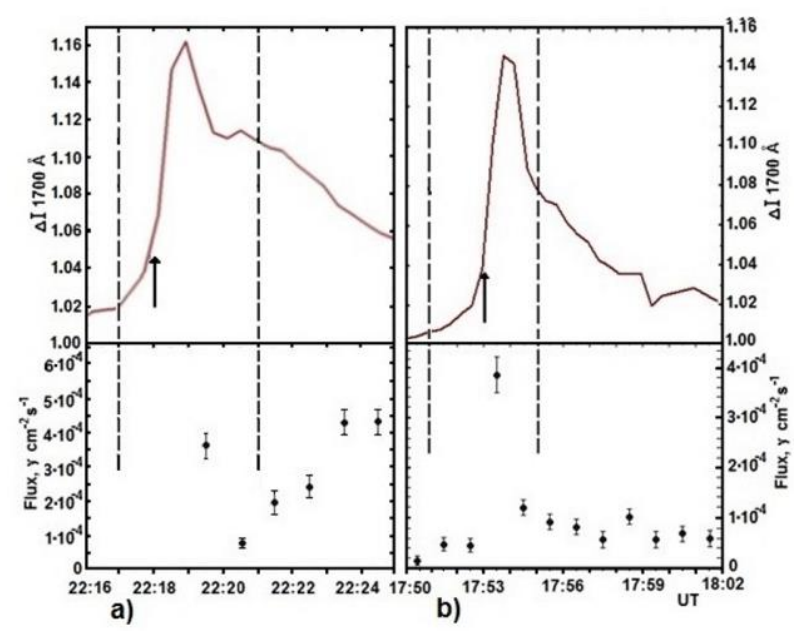

Figure 2. Variation in the relative intensity of the photospheric bright flare emission (top) and $>100 \mathrm{MeV}$ gamma-quanta (bottom) in the September 6, 2011 X2.1 flare (N13 W18) (a) and in the June 3, 2012 M3.3 flare (N15 E38) (b). Flare impulsive phase: 22:17-22:21 UT (a); 17:51-17:55 UT $(b)$. Onset of CME (vertical arrow): 22:18 UT $(a)$; 17:53 UT $(b)$. Vertical dashed lines indicate time limits of the flare impulsive phase

Table 1

Characteristics of events of interest as derived from SC observations

\begin{tabular}{|c|c|c|c|c|c|}
\hline $\begin{array}{c}\text { Date } \\
\text { of flare }\end{array}$ & $\begin{array}{c}\text { X-ray im- } \\
\text { portance } \\
\text { flare coordi- } \\
\text { nates }\end{array}$ & $\begin{array}{c}\text { UT of onset and } \\
\text { end } \\
\text { of impulsive } \\
\text { phase }\end{array}$ & $\begin{array}{c}\text { UT of onset of } \\
\text { CME } \\
V, \mathrm{~km} / \mathrm{s}\end{array}$ & $\begin{array}{c}\text { Energy } \\
\text { of hard } \\
\text { X-rays, } \mathrm{keV}\end{array}$ & $\begin{array}{c}F_{\gamma>100 \mathrm{MeV}}^{\mathrm{max}} \\
\mathrm{cm}^{-2} \mathrm{~s}^{-1}\end{array}$ \\
\hline 1 & 2 & 3 & 4 & 5 & 6 \\
\hline $\begin{array}{c}\text { August 09, } \\
2011\end{array}$ & X6.9 N16W70 & $08: 00-08: 05$ & $08: 02,1610$ & $800-7000$ & $7.7 \cdot 10^{-4}$ \\
\hline $\begin{array}{c}\text { September 06, } \\
2011\end{array}$ & X2.1 N14W18 & $22: 17-22: 21$ & $22: 18,1000$ & $300-1000$ & $3.5 \cdot 10^{-4}$ \\
\hline June 03, 2012 & M3.3 N15W38 & $17: 51-17: 55$ & $17: 53,892$ & $300-1000$ & $3.8 \cdot 10^{-4}$ \\
\hline $\begin{array}{c}\text { October 11, } \\
2013\end{array}$ & M1.9 N21E101 & $07: 08-07: 24$ & $07: 08,1200$ & - & $4.7 \cdot 10^{-4}$ \\
\hline $\begin{array}{c}\text { September 01, } \\
2014\end{array}$ & X2.1 N14E126 & $10: 57-11: 13$ & $10: 57,2000$ & $310-1180$ & $4.8 \cdot 10^{-3}$ \\
\hline $\begin{array}{c}\text { September 10, } \\
2017\end{array}$ & X8.2 S08W88 & $15: 50-16: 06$ & $15: 51,3163$ & $500-2000$ & $1.3 \cdot 10^{-2}$ \\
\hline
\end{tabular}

The end of the flare impulsive phase (Table 1, column 3 ) is determined by the time when the derivative of $\mathrm{X}$-ray flux $d F_{\mathrm{X}} / d t$ in the region (1-8) $\AA$ becomes zero at transition from rise to fall on the $\mathrm{X}$-ray emission plot (see Figure 3 ). Then, with negative $d F_{\mathrm{X}} / d t$, the flare emission main phase begins to develop. The time interval between the maximum of the emission flux derivative $d F_{\mathrm{X}} / d t$ and the onset of the main phase is considered to be the explosive stage of the impulsive phase. It is assumed that in this period the flare plasma in the photosphere and chromosphere begins to quickly rise and fill the entire volume of coronal arcs, causing their maximum emission.

The initial moments of CME development (Table 1, column 4), presented in the SOHO/LASCO catalog, have been specified via SDO/AIA filtergrams [Share et al., 2017a].

The electrons accelerated during energy dissipation in the current sheet are sources of bremsstrahlung radiation in a quantum energy range from $300 \mathrm{keV}$ to $7 \mathrm{MeV}$ (as observed by RHESSI and Fermi/GBM). These energy values are typical for impulsive phases of flares of different power. For the October 11, 2013 event there is no data owing to the presence of an active region behind the east limb.

For the events of interest in the flare impulsive phase, we have compared moments of the beginning of CME development, maxima in changes of the relative intensity of flare photospheric emission, and enhancement of $>100 \mathrm{MeV}$ gamma-quanta. We used Fermi data on gamma-ray emission with 1-min resolution, presented in [Share et al., 2017a, b; Omodei et al., 2018]. 


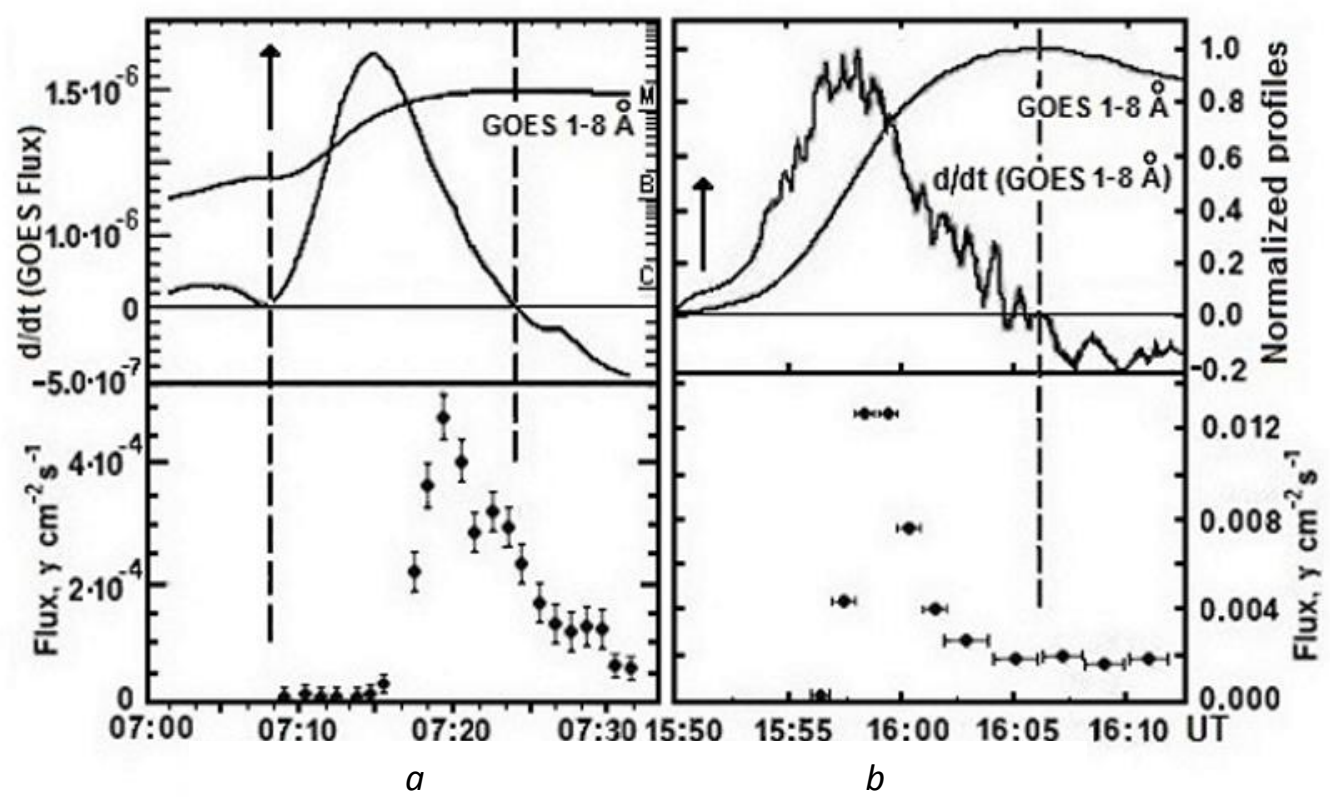

Figure 3. October 11, 2013 M1.9 event (N21, E101) (a) and September 10, 2017 X8.2 event (S08, W88) (b). Flare impulsive phase: 07:08-07:24 UT $(a) ; 15: 50-16: 06$ UT $(b)$. Onset of CME (vertical arrow):07:08 UT $(a)$; 15:51 UT $(b)$. Vertical dashed lines indicate time limits of the flare impulsive phase. X-ray flux in a range 1-8 $\AA$ and its time derivative $d F_{\mathrm{X}} / d t$ (top, normalized curves); gammaquanta with energies of $>100 \mathrm{MeV}$ (bottom)

A feature of the August 9, 2011 event is that the onset of CME (at 08:02 UT) coincides with the beginning of the maximum impulsive phase of the flare, which causes particle acceleration and plasma heating during dissipation in current sheets - magnetic reconnection regions in the active region structure. The flare impulsive phase concurs with both magnetic reconnection and additional proton acceleration at the CME shock front (typical process in the main phase of sustained proton events). Such a feature of development is also typical for the rest of the events considered (see Figures below).

Since the active region that produced the October 11, 2013 flare was at the E101 heliolongitude behind the apparent east solar limb, it was impossible to construct a time profile of the relative intensity of the bright photospheric emission during the flare development. Figure 3 therefore shows a curve of X-ray evolution in the (1-8) Å region and its time derivative $d F_{\mathrm{X}} / d t$ (SDO data) whose zero value defines the boundary between the impulsive and main phases of the flare event. As has been shown previously [Minasyants et al., 2018], the X-ray flux derivative in the impulsive phase correlates with the relative intensity of flare plasma at $\lambda 1700 \AA$. For the October 11, 2013 event due to the sustained CME interaction with the flareinduced stream, we could observe time variations of $F_{\gamma>100 \mathrm{MeV}}$ in more detail.

Omodei et al. [2018] have compared time variations in $>100 \mathrm{MeV}$ gamma-quanta and spectral index of protons for the September 10, 2017 event. The observed similarity in the behavior of these parameters confirms that protons play a major role in generating the high-energy gamma-ray emission in the flare impulsive phase.

The very high velocity of the CME shock front on September 10, 2017 undoubtedly affected the amount of gamma flux. We recorded a flux enhancement
$F_{\gamma>100 \mathrm{MeV}}$ by almost two orders of magnitude as compared to other events (Table 1, column 6). The flux appeared to be maximum $F_{\gamma>100 \mathrm{MeV}}^{\max }=0.013 \mathrm{~cm}^{-2} \mathrm{~s}^{-1}$ for the entire period of solar observations with the Fermi/LAT telescope.

A feature of the September 1, 2014 event (Figure 4) is the location of the active region within $40^{\circ}$ behind the east solar limb. The data on the development of the flare and CME was acquired by SC that monitored this region behind the limb. The soft X-ray profiles as well as X-ray importance of the flare (according to the GOES classification) were determined from MESSENGER SAX observations. The time of onset of the flare emission was determined and its further development was described using STEREO $\lambda$ $195 \AA$ filtergrams. The data on X-rays in an energy range from 20 to $1180 \mathrm{keV}$ was obtained by WIND/KONUS together with Fermi/GBM [Ackermann et al., 2017].

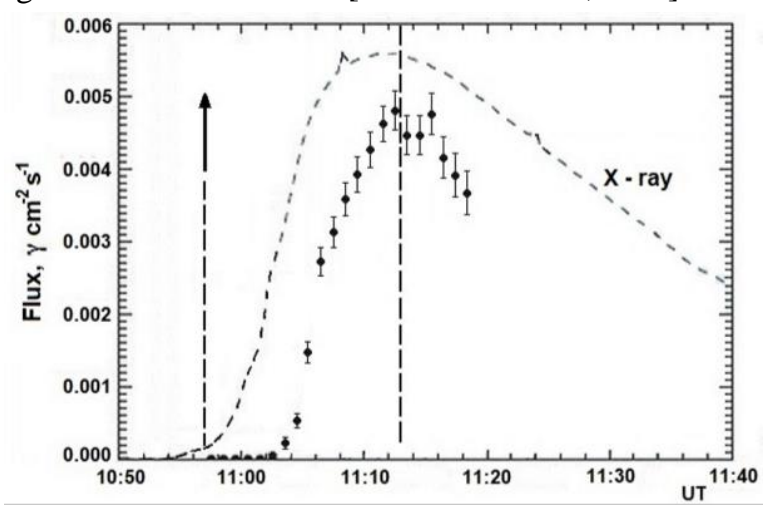

Figure 4. Variations in soft X-rays and $>100 \mathrm{MeV}$ gamma-quanta during the September 1, 2014 X2.1 event (N14 E126). Flare impulsive phase: 10:57-11:13 UT. Onset of CME (vertical arrow): 10:57 UT. Vertical dashed lines indicate boundaries of the flare impulsive phase 


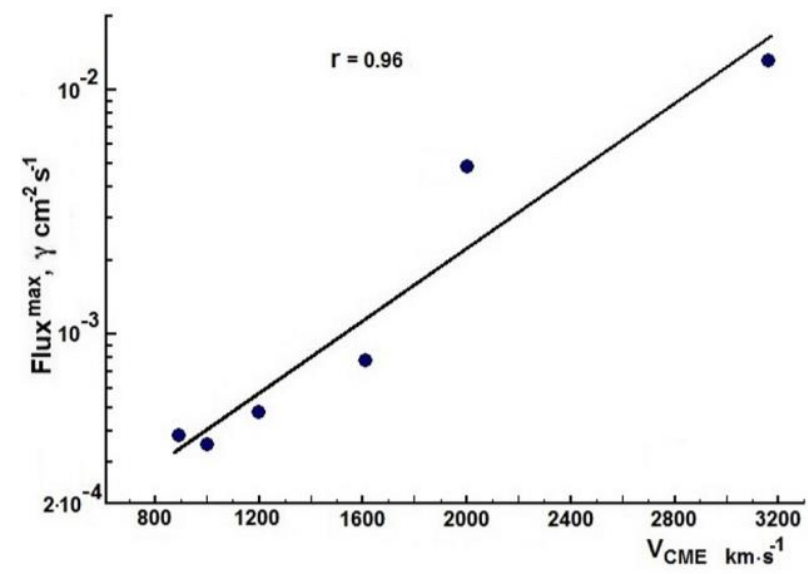

Figure 5. Maximum gamma fluxes $F_{\gamma>100 \mathrm{MeV}}^{\max }$ versus

CME linear velocity accompanying the development of flares when observed with the LAT instrument at 1-min resolution throughout the operating period of the Fermi telescope

We have compared maximum gamma fluxes $F_{\gamma>100 \mathrm{MeV}}^{\max }$ in flare impulsive phases with CME velocities during their simultaneous development (Figure 5). The calculated Pearson correlation coefficient $r=0.96$.

We have determined time intervals between the onset of CME in the active region and the onset of the flare impulsive phase. In most cases, CME was preceded by the onset of the flare impulsive phase. It is noteworthy that for the six events in which the $>100 \mathrm{MeV}$ gamma fluxes at the beginning of the flare impulsive phase were recorded, these interval (in minutes) was +2 , $+1,+2,0,0,+1$.

Only in three events - March 7, 2012, February 25, 2014, and September 6, 2011, maximum gamma fluxes were recorded $F_{\gamma>100 \mathrm{MeV}}^{\max }$ in the flare main phase within values typical of the impulsive phase of the flare under study. We have compared results for 30 events with $F_{\gamma>100 \mathrm{MeV}}$ obtained from 1-min resolution observations with the LAT instrument throughout the operating period of the Fermi telescope. During the rest of the events there were lower maximum gamma fluxes $F_{\gamma>100 \mathrm{MeV}}^{\max }$ in the flare main phase.

\section{INFLUENCE OF DIFFERENT PARAMETERS ON MAXIMUM GAMMA FLUXES OF >100 MeV DURING THE FLARE DEVELOPMENT}

We have presented 1-min resolution profiles for the periods of maximum development of $>100 \mathrm{MeV}$ gamma fluxes (Fermi) [Share et al., 2017a, b] for 17 of 32 flare events. Using this data, we compared maximum gamma fluxes with parameters that may have an effect on $F_{\gamma>100 \mathrm{MeV}}^{\max }$ in both the flare impulsive and main phases.

In column 3 of Table 2 is X-ray importance of flares, according to the GOES classification, time of their onset and end. By definition, the time of end of a GOES Xray flare is the time when the flux between the maximum value and the background level before the flare decreases by a factor of two.
Maximum gamma fluxes $F_{\gamma>100 \mathrm{Mev}}^{\max }$ and times of their recording are shown in columns 4 and 5 respectively. We also determined the time difference between recording of $F_{\gamma>100 \mathrm{MeV}}^{\max }$ and end of the flare from X-rays (GOES) for each event (column 7).

The study of interactions of CME, flare processes, and shock waves, which are accompanied by generation of high-energy gamma-ray emission with quantum energies above $100 \mathrm{MeV}$, enabled us to evaluate the effectiveness of these interactions during the development of active processes.

Figure 6 identifies two different groups of values: events with higher $F_{\gamma>100 \mathrm{MeV}}^{\max }$ and sharp increases in fluxes with increasing CME velocity (red dots); events with opposite characteristics (black dots). Note that in most of the events the CME linear velocities in both the groups have close values. This fact points to the existence of a parameter effectively influencing the increase in the gamma flux.

The development of flares with X-ray importance from M1 to X3 also shows that this parameter does not play a leading part in developing gamma fluxes.

The comparison made in Figures 6 and 7 suggests that to high values of $F_{y>100 \mathrm{Mev}}^{\max }$ (red dots) correspond low CME velocities and low X-ray importance of flares (black dots).

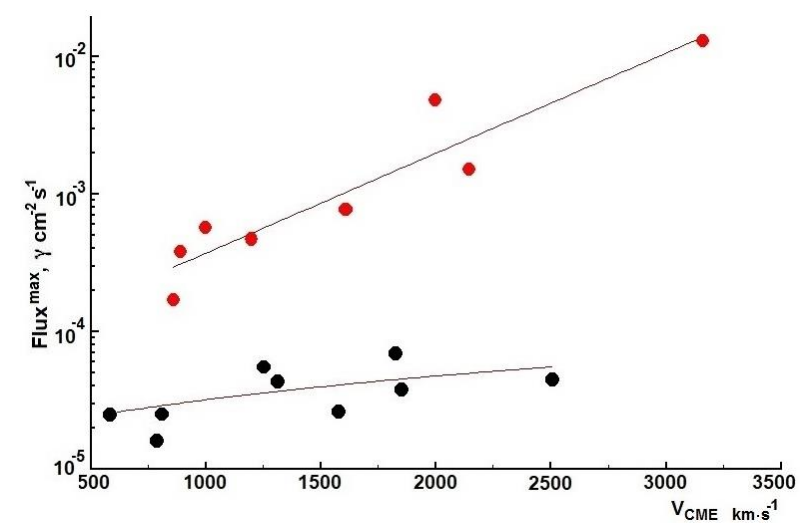

Figure 6. Maximum gamma fluxes of $>100 \mathrm{MeV}$ versus CME linear velocity for 17 flare events

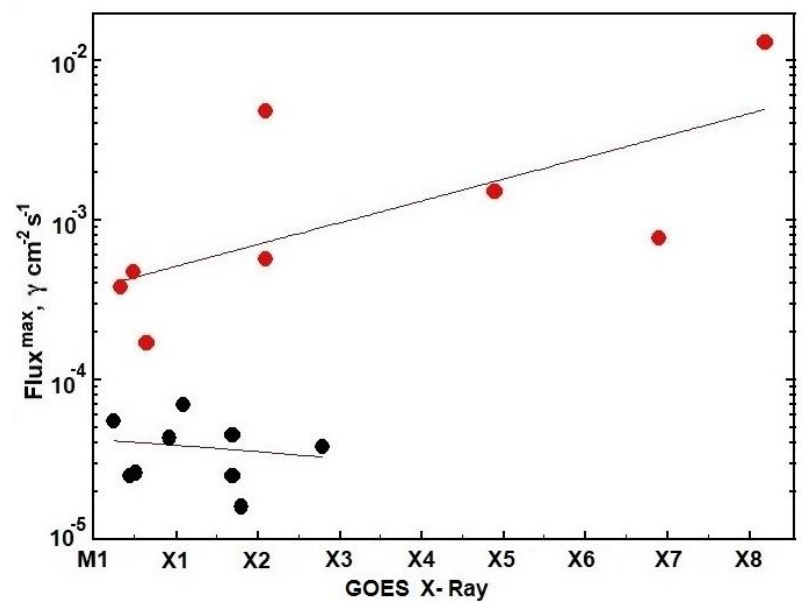

Figure 7.Maximum gamma fluxes of $>100 \mathrm{MeV}$ versus $\mathrm{X}$ ray importance (GOES) of flares for 17 events 
Table 2

Characteristics of flare events with $F_{\gamma>100} \mathrm{MeV}$

\begin{tabular}{|r|l|c|c|c|c|c|}
\hline $\mathrm{N}$ & \multicolumn{1}{|c|}{$\begin{array}{c}\text { Date, } \\
\text { coordinates }\end{array}$} & $\begin{array}{c}\text { X-ray importance } \\
\text { moments of onset } \\
\text { and end (GOES) }\end{array}$ & $F_{\gamma>100 \mathrm{Mev}}^{\max }, \mathrm{cm}^{-2} \mathrm{~s}^{-1}$ & $\begin{array}{c}\text { Time } \\
F_{\gamma>100 \mathrm{Mev}}^{\max }\end{array}$ & $\begin{array}{c}\text { CME } \\
\text { velocity } \\
V, \\
\mathrm{~km} / \mathrm{s}\end{array}$ & $\begin{array}{c}\Delta t \text {, } \\
\text { min }\end{array}$ \\
\hline 1 & \multicolumn{1}{|c|}{2} & 3 & 4 & 5 & 6 & 7 \\
\hline 1 & June 07, 2011, S21W54 & $\mathrm{M} 2.5,06: 16-06: 59$ & $5.5 \cdot 10^{-5}$ & $08: 02$ & 1255 & $01: 03$ \\
\hline 2 & August 04, 2011, N19W46 & $\mathrm{M} 9.3,03: 41-04: 04$ & $4.3 \cdot 10^{-5}$ & $05: 05$ & 1315 & $01: 01$ \\
\hline 3 & August 09, 2011, N16W70 & $\mathrm{X} 6.9,07: 48-08: 08$ & $7.7 \cdot 10^{-4}$ & $08: 03$ & 1610 & $-00: 05$ \\
\hline 4 & September 06, 2011, N14W18 & $\mathrm{X} 2.1,22: 12-22: 24$ & $5.7 \cdot 10^{-4}$ & $22: 27$ & 1000 & $00: 03$ \\
\hline 5 & September 07, 2011, N18W32 & $\mathrm{X} 1.8,22: 32-22: 44$ & $1.6 \cdot 10^{-5}$ & $23: 37$ & 792 & $00: 53$ \\
\hline 6 & January 27, 2012, N35W81 & $\mathrm{X} 1.7,17: 37-18: 56$ & $4.5 \cdot 10^{-5}$ & $19: 48$ & 2508 & $00: 52$ \\
\hline 7 & May 17, 2012, N05W77 & $\mathrm{M} 5.1,01: 25-02: 14$ & $2.6 \cdot 10^{-5}$ & $02: 38$ & 1582 & $00: 24$ \\
\hline 8 & June 03, 2012, N15E38 & $\mathrm{M} 3.3,17: 48-17: 57$ & $3.8 \cdot 10^{-4}$ & $17: 54$ & 892 & $-00: 03$ \\
\hline 9 & July 06, 2012, S17W52 & $\mathrm{X} 1.1,23: 01-23: 14$ & $6.9 \cdot 10^{-5}$ & $23: 26$ & 1828 & $00: 12$ \\
\hline 10 & April 11, 2013, N07E13 & $\mathrm{M} 6.5,06: 55-07: 29$ & $1.7 \cdot 10^{-4}$ & $07: 13$ & 861 & $-00: 16$ \\
\hline 11 & May 13, 2013, N10E80 & $\mathrm{X} 2.8,15: 48-16: 16$ & $3.8 \cdot 10^{-5}$ & $17: 42$ & 1850 & $01: 26$ \\
\hline 12 & October 11, 2013, N21E103 & $\mathrm{M} 4.9,07: 01-07: 45$ & $4.7 \cdot 10^{-4}$ & $07: 19$ & 1200 & $-00: 26$ \\
\hline 13 & October 25, 2013, S08E71 & $\mathrm{X} 1.7,07: 53-08: 09$ & $2.5 \cdot 10^{-5}$ & $08: 17$ & 587 & $00: 08$ \\
\hline 14 & October 28, 2013, S14E28 & $\mathrm{M} 4.4,15: 07-15: 21$ & $2.5 \cdot 10^{-5}$ & $15: 45$ & 812 & $00: 24$ \\
\hline 15 & February 25, 2014, N00E78 & $\mathrm{X} 4.9,00: 39-01: 03$ & $1.5 \cdot 10^{-3}$ & $01: 22$ & 2147 & $00: 19$ \\
\hline 16 & September 01, 2014, N14E126 & $\mathrm{X} 2.1,10: 58-11: 34$ & $4.8 \cdot 10^{-3}$ & $11: 12$ & 2000 & $-00: 22$ \\
\hline 17 & September 10, 2017, S08W88 & $\mathrm{X} 8.2,15: 35-16: 31$ & $1.3 \cdot 10^{-2}$ & $15: 58$ & 3163 & $-00: 33$ \\
\hline
\end{tabular}

The time dependence of maximum gamma fluxes on the end of X-ray flare is, however, much in evidence. The vertical dashed line in Figure 8 separates groups with high (red dots) and lower (black dots) maximum gamma fluxes of $>100 \mathrm{MeV}$. Only one event on February 25, 2014 does not fit the overall picture. In this event, the weakened effect of the flare (X4.9) is likely to be compensated by the contribution of the CME high-speed shock front $\left(V_{\mathrm{CME}}=2147 \mathrm{~km} / \mathrm{s}\right)$, and the joint action of the flare and CME led to the increased flux value $F_{\gamma>100 \mathrm{Mev}}^{\max }$.

For each group of events we can determine mean values of $\Delta t$ between moments of maximum gamma fluxes and the time of end of the X-ray flare: $-10 \mathrm{~min}$ (Group 1) and +43 min (Group 2). Thus, the most efficient particle acceleration at the CME shock front occurs during the simultaneous development of flare and CME.

Let us give an illustrative example of the effect of $\Delta t$ on $F_{\gamma>10 \mathrm{Mev}}^{\max }$ :

- January 27, 2012 flare: $V=2508 \mathrm{~km} / \mathrm{s}, \mathrm{X} 1.7$, $\Delta t=+52 \mathrm{~min}, F_{y>100 \mathrm{Mev}}^{\max }=4.5 \cdot 10^{-5} \mathrm{~cm}^{-2} \mathrm{~s}^{-1}$;

- October 11, 2013 flare: $V=1200 \mathrm{~km} / \mathrm{s}, \mathrm{M} 4.9$, $\Delta t=-26 \mathrm{~min}, F_{\gamma>100 \mathrm{MeV}}^{\max }=4.7 \cdot 10^{-4} \mathrm{~cm}^{-2} \mathrm{~s}^{-1}$.

Thus, we have revealed the existence of two separate groups of $F_{\gamma>100 \mathrm{Mev}}^{\max }$ (Figure 6). Each group comprises events that differ in efficiency of interaction of flare particles with CME shock waves.

\section{MAIN RESULTS AND CONCLUSIONS}

In this paper, we have analyzed the evolution of

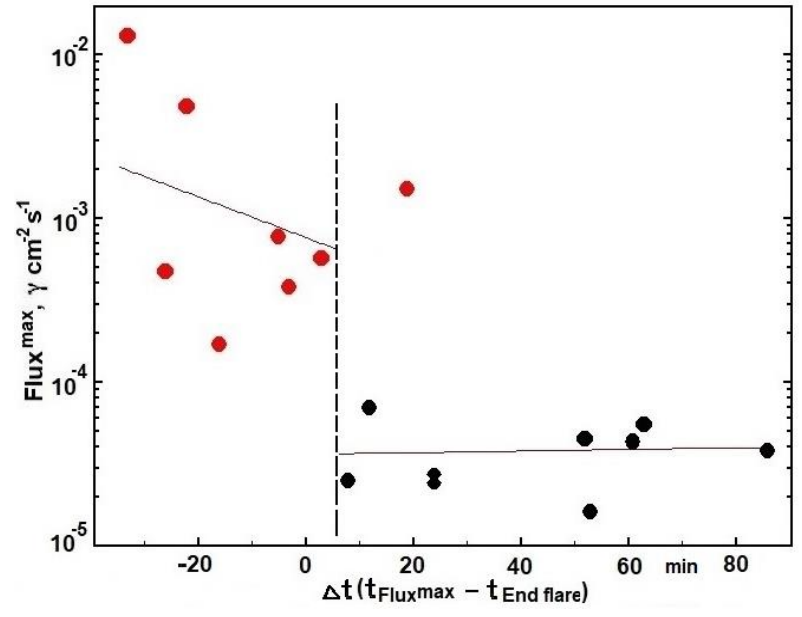

Figure 8. Maximum gamma fluxes of $>100 \mathrm{MeV}$ for each of the 17 events when compared with the time difference between $F_{p>100 \mathrm{Mev}}^{\max }$ and end of the X-ray flare (GOES)

sustained gamma fluxes at different stages of the flare process, using Fermi/LAT observations with 1-min resolution [Share et al., 2017a, b].

We focus on the determination of various laws of interaction between flare processes and CME. We have found that the time of occurrence of CME is closely related to the development of the flare impulsive phase. The most efficient particle acceleration at the CME shock front, which generates high-energy (>100 MeV) gamma fluxes, is observed when the ejection occurs and develops just before the flare impulsive phase or during this phase. Of the six events considered, only for the September 6, 2011 flare the maximum value of $F_{r>100 \mathrm{MeV}}$ 
in the impulsive phase was slightly lower than that in the main phase of the flare. In other cases, $F_{\gamma>100 \mathrm{Mev}}^{\max }$ during the impulsive phase was the highest throughout the flare event (Figures 1-4).

Note an interesting feature - the flare flux during the impulsive phase interacted with high-speed CME within a rather limited time interval - from 2 to 16 min. In the above events, we have found a direct relationship between $F_{\gamma>100 \mathrm{MeV}}^{\max }$ and CME velocity.

During the development of CME accompanied by the formation of shock waves, the energetic particle acceleration generally occurs during the main phase of the sustained gamma flare. In some cases, however, the energetic particle acceleration at the CME shock front takes place exactly in the short impulsive phase.

For 17 events we have compared $F_{\gamma>10 \mathrm{Mev}}^{\max }$ with different physical parameters in order to identify the most significant of them for generation of $>100 \mathrm{MeV}$ gammaquanta.

Presumably, the most efficient particle acceleration occurs during the simultaneous development of flare and CME. Each of the eight events with $F_{\gamma>100 \mathrm{Mev}}^{\max }$ from $1.7 \cdot 10^{-4}$ to $1.3 \cdot 10^{-2} \mathrm{~cm}^{-2} \mathrm{~s}^{-1}$ is characterized by the presence of the interval of simultaneous development of the flare process and its related CME with a shock wave (Figure 8).

The dominant effect is pronounced on the background of $F_{\gamma>100 \mathrm{MeV}}^{\max }<7 \cdot 10^{-5} \mathrm{~cm}^{-2} \mathrm{~s}^{-1}$ observed in time intervals over $20 \mathrm{~min}$ after the end of the X-ray flare at close CME velocities and X-ray importance of the flares. A decrease in the gamma-ray emission outside the interval of the joint action of the flare processes and $\mathrm{CME}$ is probably due to the fact that the proton acceleration occurs largely at the CME shock front.

Thus, during sustained events with high-energy gamma-ray emission, the most efficient particle acceleration (with $E_{\mathrm{k}}>500 \mathrm{MeV}$ ) is likely to occur if a flare and a CME shock wave develop almost simultaneously.

We express gratitude to the team of scientists in charge of experiments conducted at Fermi, SDO, GOES, SOHO, RHESSI, STEREO, MESSENGER, WIND/KONUS for providing experimental data published on the Internet.

The work was performed with budgetary funding of Program BR05336383 of the MDAI Aerospace Committee of the Republic of Kazakhstan and Basic Research Program II.16 (project II.16.3.1).

\section{REFERENCES}

Ackermann M., Allafort A., Baldini L., Barbiellini G., Bastieri D., Bellazzini R., et al. Fermi-LAT observations of high-energy behind-the-limb solar flares. arXiv:1702.00577v1 [astro-ph.SR] 2 Feb 2017. 14 p.

Akimov V.V., Afanassyev V.G., Belousov A.S., Blokhintsev I.D., Kalinkin L.F., Leikov N.G., et al. Observation of high energy gamma-rays from the Sun with the GAMMA-1 telescope $(E>30 \mathrm{MeV})$. Proc. $22^{\text {nd }}$ ICRC. 1991, vol. 3, pp. 73-76.
Altyntsev A.T., Banin V.G., Kuklin G.V., Tomozov V.M. Solnechnye vspyshki [Solar Flares]. Moscow, Nauka Publ., 1982. 246 p. (In Russian).

Golovko A.A., Kuklin G.V., Mordvinov A.V., Tomozov V.M. The role of shear motions in the production of a preflare situation. Contributions of the Astronomical Observatory Skalnate Pleso. 1986, vol. 15, pp. 243-250.

Gopalswamy N., Mäkela P., Yashiro S., Lara A., Xie H., Akiyama S., MacDowall R.J. Interplanetary type II radio bursts from Wind/WAVES and sustained gamma-ray emission from Fermi/LAT: evidence for shock source. Astrophys. J. Lett. 2018., vol. 868, L19, 8 p. DOI: 10.3847/2041-8213/aaef36.

Gopalswamy N., Mäkela P., Yashiro S., Lara A., Xie H., Akiyama S., MacDowall R.J. Fermi, Wind and SOHO observations of sustained gamma-ray emission from the Sun. URSI AP-RASC 2019, New Delhi, India, 09-15 March 2019. URL: https://arxiv.org/ftp/arxiv/papers/1810/1810.08958.pdf (accessed 01.04.2019).

Grechnev V.V., Kurt V.G., Chertok I.M., Uralov A.M., Nakajima H., Altyntsev A.T., Belov A.V., Yushkov B.Yu., Kuznetsov S.N., Kashapova L.K., Meshalkina N.S., Prestage N.P. An extreme solar event of 20 January 2005: properties of the flare and the origin of energetic particles. Solar Phys. 2008, vol. 252, pp.149-177. DOI: 10.1007/s11207-008-9245-1.

Knizhnik K.J., Antiochos S.K., DeVore C.R., Wyper P.F. The mechanism for the energy buildup driving solar eruptive events. Astrophys. J. Lett. 2017, vol. 851, L17, 6 p. DOI: 10.3847/2041-8213/aa9e0a.

Kurt V.G., Yushkov B.Yu., Kudela K., Galkin V.I. Highenergy gamma emission of solar flares as an indicator of acceleration of high-energy particles. Proc. $31^{\text {st }}$ National Conference on Cosmic Rays. Moscow, MSU, 2010, pp. 1-5. (In Russian).

Li Y., Sun X., Ding M.D., Qiu J., Priest E.R. Imaging observations of magnetic reconnection in a solar eruptive flare. Astrophys. J. 2017, vol. 835, 190, 8 p. DOI: $10.3847 / 1538-$ 4357/835/2/190.1.

Livshits M.A. Solar flares: observation results and gasdynamic processes. Plazmennaya geliofizika [Plasma Heliophysics]. Moscow, Nauka Publ., 2008, vol. 1, pp. 60-81. (In Russian).

Manchester W., Kilpua K.J., Liu Y.D., Lugaz N., Riley P., Török T., Vršnak B. The physical processes of CME/ICME evolution. Space Sci. Rev. 2017, vol. 212, pp. 1159-1219. DOI: $10.1007 / \mathrm{s} 11214-017-0394-0$.

Minasyants G.S., Minasyants T.M., Tomozov V.M. Features of the development of gamma-rays in a solar flare February 25, 2014. News National Academy RK, Phys.-Math. Ser. 2018, vol. 4, no. 320, pp. 15-21.

Murphy R.J., Dermer C.D., Ramaty R. High-energy processes in solar flares. Astrophys. J. Suppl. 1987, vol. 63, pp. 721-748.

Omodei N., Pesce-Rollins M, Longo F., Allafor A., Krucker S. Fermi-LAT observations of the 2017 September $10^{\text {th }}$ solar flare. arXiv: 1803.07654v1 [astro-ph.HE]. 2018, 6 p.

Priest E.R., Forbs T. Magnitnoe peresoedinenie. Magnitogidrodinamicheskaya teoriya i prilozheniya [Magnetic Reconnection. Magnetohydrodynamic Theory and Applications]. Moscow, Fizmatlit Publ., 2005. 591 p. (In Russian).

Share G.H., Murphy R.J., Tolbert A.K., Dennis B.R., White S.M., Schwartz R.A., Tylka A.J. Characteristics of sustained $>100 \mathrm{MeV}$ ray-emission associated with solar flares. arXiv:1711.01511v1 [astro-ph.SR]. 2017a, 83 p.

Share G.H., Murphy R.J., Tolbert A.K., Dennis B.R., White S.M., Schwartz R.A., Tylka A.J. Characteristics of thirty second-stage $>100 \mathrm{MeV} \gamma$-ray events accompanying solar flares. ApJS in review, arXiv 1711.01511v1. 2017b, 34 p.

Shibata K. Reconnection model of flares. Solar physics with radio observations. Proc. of Nobeyama Symposium. 1998, 
pp. 381-389. (NRO Report N 479).

Somov B.V. Plasma Astrophysics: Reconnection and Flares. New York, Springer, 2013, 504 p. DOI: 10.1016/ S02731177(97)00968-X.

Tylka A.J., Share G.H., Dietrich W.F., Murphy R.J., Ng C.K., Shea M.A., Smart D.F. Solar protons above $500 \mathrm{MeV}$ in the Sun's atmosphere and in interplanetary space. Report EGU Gener Assembly, Vienna, Austria 27 April — 02 May 2014. 201 EGU2014-16847, 41 p.

How to cite this article

Minasyants G.S., Minasyants T.M., Tomozov V.M. Features of development of sustained fluxes of high-energy gamma-ray emission at different stages of solar flares. Solar-Terrestrial Physics. 2019. Vol. 5. Iss. 3. P. 10-17. DOI: 10.12737/stp-53201902. 